\title{
Gynura segetum-induced liver injury leading to acute liver failure: a case report and literature review
}

\author{
Zilong Zhang 1,2, Haibo Zou ${ }^{1,2}$, Zonglin Dai ${ }^{1,2}$, Jin Shang ${ }^{1,2}$, Shining Sure ${ }^{2,3}$, Chunyou Lai ${ }^{2}$, Ying Shi ${ }^{1,2}$, \\ Qinyan Yang ${ }^{2}$, Guangming Xiang ${ }^{2}$, Yutong Yao ${ }^{1,2}$, Tianhang Feng ${ }^{1,2}$, Deyuan Zhong ${ }^{1,2}$ and Xiaolun Huang ${ }^{1,2^{*}}$ (D)
}

\begin{abstract}
Background: Gynura segetum (GS) is widely used in medical care and in community settings in China as the herbal remedy. It is widely thought to have antiphlogistic properties and pain relief in traditional Chinese medicine. It has been reported that GS can cause chronic drug-induced liver injury (DILI), manifested as hepatic sinusoid obstruction syndrome (HOSO). But case reports of acute DILI developing acute liver failure (ALF) due to GS are extremely rare.
\end{abstract}

Case presentation: We report a case of a 63-year-old female patient with hepatolithiasis for more than 6 years. There were no deterioration of liver function and no history of viral liver disease, autoimmune liver disease, blood transfusion or surgical allergy before operation. ALF and grade II liver encephalopathy occurred after partial hepatectomy. To follow up the medical history, the patient has been taking GS (Tusanqi) for a year and a half. The causality assessment was done by the updated Roussel Uclaf Causality Assessment Method, and the possibility of DILI caused by GS as highly probable for the score was 6 points. Excluding other causes, a diagnosis of DILl-associated ALF was established. After symptomatic support and artificial liver support system (ALSS) treatment, the clinical symptoms and signs of the patients were significantly improved. After discharge, the liver function of the patients returned to normal.

Conclusions: Based on this rare case of severe liver injury, we recommend that timely prevention, identification, and appropriate management of DILI is essential for patients with a history of taking GS and other hepatotoxic drugs, and careful monitoring of liver function for patients with DILI could avoid ALF as far as possible.

Keywords: Gynura segetum, Drug-induced liver injury, Acute liver failure, Case report

\section{Background}

Gynura segetum (Tusanqi or Jusanqi) is an erroneous species substitute of the Traditional Chinese medicines herb Sedum aizoon, and it has been widely used in Chinese folk medicine and plays a role in promoting microcirculation and relieving pain. However, its hepatic

\footnotetext{
*Correspondence: huangxiaolun@med.uestc.edu.cn

1 Department of Hepatobiliary-Pancreatic Surgery, Cell Transplantation Center, Sichuan Provincial People's Hospital, University of Electronic Science and Technology of China, Chengdu, China

Full list of author information is available at the end of the article
}

toxicity is often overlooked, and it has been reported that GS could cause DILI [1-3].

GS-induced DILI involves fibrous obliteration and destruction of the central veins and sub-lobular venules, leading to cirrhosis [3]. The clinical characteristics of GSinduced DILI are not typical, its diagnosis is very difficult and is mainly based on a careful history and exclusion of other causes. A few cases of DILI relating to GS usage have been reported in literature. However, GS-induced DILI developing into ALF has not been reported. The diagnostic strategy, management, and prognosis of GSinduced DILI are rarely described. 
In this case report, we report a case of a patient with severe GS-induced DILI who developed ALF and was successfully resuscitated by ALSS and reviewed the papers related to GS-induced DILI in the literature.

\section{Case presentation}

A 63-year-old woman with a history of hepatolithiasis for more than six years was suffering from intermittent low back pain. She was followed up in a local hospital for a long time, though the treatment was not effective. The patient was reexamined in the local hospital 20 days ago. The upper abdominal enhanced CT revealed intrahepatic bile duct stones with extrahepatic bile duct dilatation, atrophy of the left lobe of the liver, cholestasis of the left lobe of the intrahepatic bile duct and no sign of portal hypertension (Fig. 1A). She was admitted to Sichuan Provincial People's Hospital for further diagnosis and treatment. Clinical history documented hypertension in the patient. The patient also denied drug use, trauma, surgery, alcohol abuse, and blood transfusion and claimed no history of other systemic diseases, no recent travel history, no mushroom ingestion, no family history of similar hepatolithiasis or any liver disease.

No obvious abnormality was reflected in her laboratory findings during admission (Table 1). Viral serological testing was reported negative for hepatitis A, hepatitis $C$, and HIV, and the patient was immune to hepatitis B. Serological screening tests for autoimmune diseases were also negative. Atrophy of the left lateral lobe of the liver, hepatolithiasis with dilatation of the bile duct, thickening of the bile duct wall was obtained from abdominal MRI (Fig. 1B). The patient was diagnosed with left hepatolithiasis, left extrahepatic lobe atrophy, and high-risk hypertension. The liver function reserve of the patient was determined to be Child-Pugh class $\mathrm{A}$. The patient voluntarily gave consent and signed a written informed consent form for elective surgery. Under general anesthesia, the patient underwent successful laparoscopic left lateral hepatectomy and bile duct exploration. Histology reported dilated bile duct epithelial hyperplasia, infiltration of inflammatory cell infiltrate, necrosis of hepatocytes, formation of the lymphoid follicle, and local liver fibrosis (Fig. 2A). The patient received smooth postoperative treatment.

The patient's liver function significantly deteriorated on the second day after the operation (Table 1), coagulation function test showed PT $38.7 \mathrm{~s}(9.8-12.1 \mathrm{~s})$, activated partial thromboplastin time $42.8 \mathrm{~s}(23.3-32.5 \mathrm{~s})$ and D-dimmer $35.33 \mathrm{mg} / \mathrm{L}$, indicating acute liver injury. Coagulation dysfunction developed following operation was managed with administration of 3 units of Freshfrozen plasma (FFP) to supplement coagulation factors. However, the patient's clinical condition progressively worsened at POD4, and the patient suddenly became manic, gibberish, and subsequently confused. Physical examination revealed severe icterus of the sclera and skin, disturbances in consciousness in the patient, and Babinski's sign was positive in the right limb. There was continuous regression in the patient's clotting function and liver biochemistry (Table 1); Additional blood tests showed lactate $5.9 \mathrm{mmol} / \mathrm{L}(0.5-1.7 \mathrm{mmol} / \mathrm{L})$, blood ammonia 328.9umol/L(18-72umol/L), $\mathrm{PH} 7.452, \mathrm{PO}_{2}$ 104.2mmh, $\mathrm{PCO}_{2} 24.2 \mathrm{mmhg}, \mathrm{HCO}_{3}^{-} 20.4 \mathrm{mmol} / \mathrm{l}$, base excess $-2.9 \mathrm{mmol} / \mathrm{l}, \mathrm{K}^{+} 2.6 \mathrm{mmol} / \mathrm{L}$. Consequently, the patient was admitted to the Intensive care unit (ICU) with the diagnosis of ALF and grade II liver encephalopathy. Symptomatic treatment included prophylactic antimicrobial therapy, FFP, human serum albumin, platelet transfusion, and nutritional liver support. For the management of hyperammonemia and hypokalemia, lactose enemas in combination with intravenous infusion of potassium chloride and a
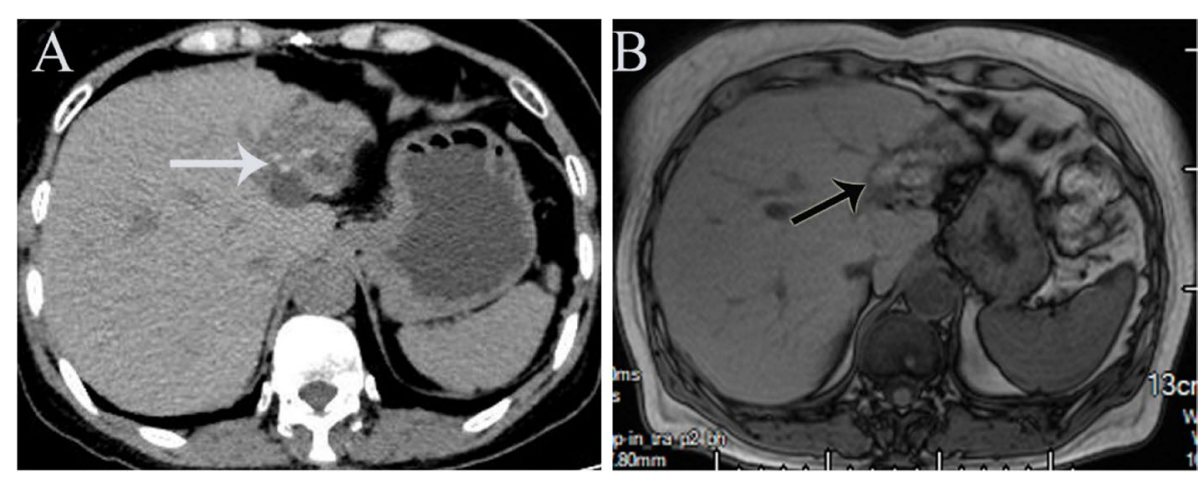

Fig. 1 Preoperative imaging workup of left intrahepatic bile duct calculi and hepatic atrophy A the upper abdominal enhanced CT showing hepatolithiasis with dilatation of right-sided intrahepatic bile ducts (white arrow). B Contrast-enhanced MRI further delineated the anatomy of atrophy of the left lateral lobe of the liver (black arrow) 
Table 1 Alteration in laboratory and serology data during treatment

\begin{tabular}{|c|c|c|c|c|c|c|}
\hline Laboratory exam & $\begin{array}{l}\text { Reference Range, } \\
\text { Adults }^{\mathrm{a}}\end{array}$ & On admission & At POD 2 & At POD 4 & $\begin{array}{l}\text { At POD } 8 \text { (After } \\
\text { ALSS) }\end{array}$ & $\begin{array}{l}\text { At POD } 21 \\
\text { (Date of } \\
\text { discharge) }\end{array}$ \\
\hline WBC (× $109 / L)$ & $3.50-9.50$ & 3.54 & 9.54 & 6.61 & 9.45 & 2.89 \\
\hline $\mathrm{HGB}(\mathrm{g} / \mathrm{L})$ & $115-150$ & 124 & 100 & 107 & 86 & 77 \\
\hline PLT (× 109 /L) & $101-320$ & 178 & 163 & 63 & 9 & 300 \\
\hline hsCRP (mg/L) & $0-5.00$ & $<0.5$ & 18.23 & 36.41 & 15.77 & 12.66 \\
\hline AST (U/L) & $13-35$ & 26 & 2743 & 4675 & 83 & 63 \\
\hline $\operatorname{ALT}(U / L)$ & $7-40$ & 18 & 2843 & 6307 & 653 & 43 \\
\hline TBIL & $0.0-23.0$ & 20.4 & 81.2 & 93.2 & 108.8 & 80.7 \\
\hline DBIL & $0.0-8.0$ & 4.9 & 34.2 & 46.7 & 34.6 & 65.7 \\
\hline$|\mathrm{B}| \mathrm{L}$ & $0.0-20.0$ & 15.5 & 47 & 46.5 & 74.2 & 15.0 \\
\hline LDH & $120-250$ & 212 & 1982 & 1606 & 482 & 249 \\
\hline $\mathrm{ALP}(\mathrm{U} / \mathrm{L})$ & 50-135 & 95 & 118 & 145 & 82 & 149 \\
\hline ALB (g/L) & $40.0-55.0$ & 43.4 & 34.3 & 33.8 & 31.3 & 26.3 \\
\hline GLU (mmol/L) & $3.90-6.10$ & 4.71 & 3.86 & 6.11 & 6.7 & 5.74 \\
\hline PT(sec) & $9.8-12.1$ & 10 & 38.7 & 31.9 & 20.9 & 14.5 \\
\hline PT\% (\%) & $70.0-130.0$ & 129.4 & 13.7 & 17.4 & 30.8 & 55.7 \\
\hline PT-INR & $0.96-1.16$ & 0.91 & 3.7 & 3.03 & 1.95 & 1.33 \\
\hline D-dimer (mg/L FEU) & $0.00-0.55$ & 0.4 & 35.33 & 27.01 & 13.17 & 4.56 \\
\hline
\end{tabular}

${ }^{a}$ Reference values are affected by many variables, including the patient population and the laboratory methods used. The ranges used at Sichuan Provincial People's Hospital are for adults
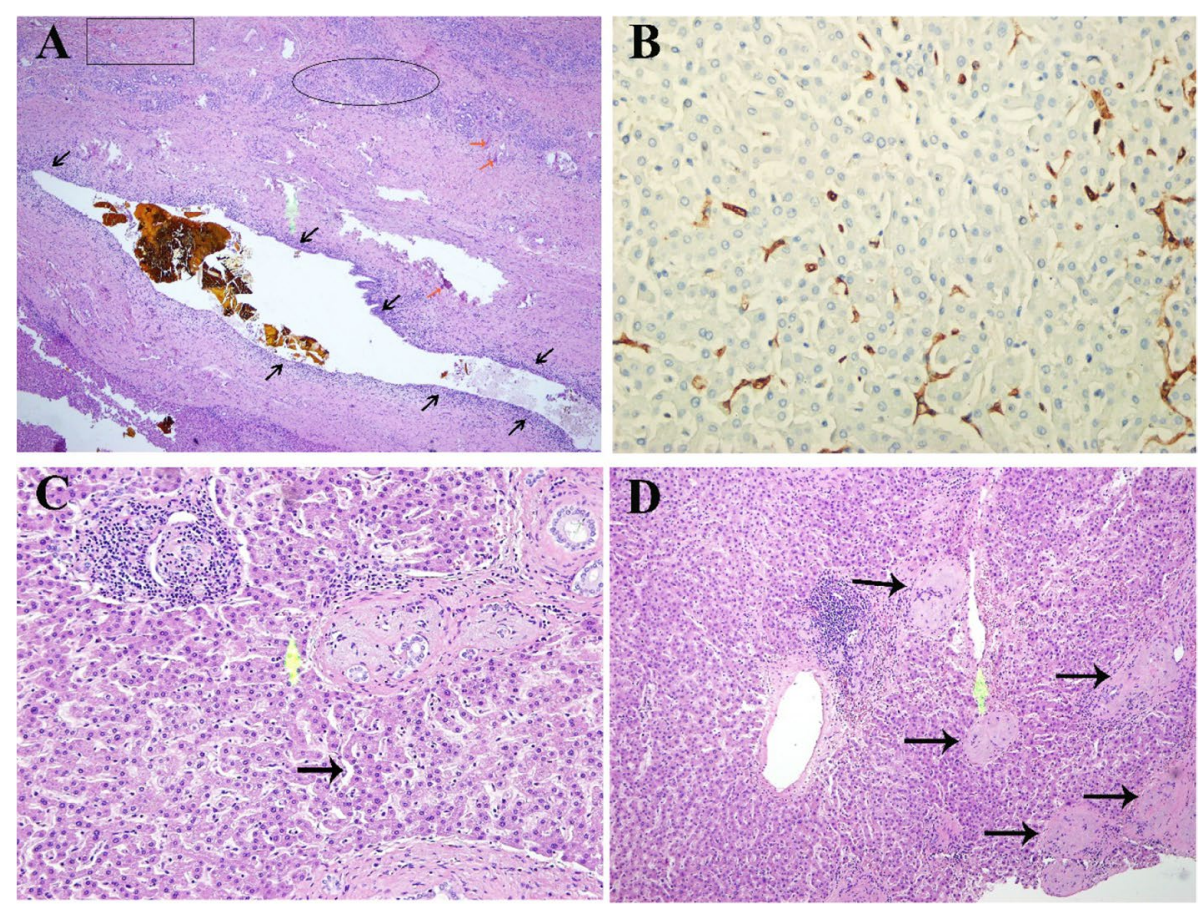

Fig. 2 Histological findings of liver biopsy specimens (A) Bile duct epithelial hyperplasia, inflammatory cell infiltration (black arrow), necrosis of hepatocytes (orange arrow), formation of the lymphoid follicle (blank irregular round), and local liver fibrosis (blank rectangle). B CD34 immunostaining of liver biopsy revealed the presence of positive CD34 in the subendothelial space of central veins and perivenular zones. C Local hepatic sinusoid was highly dilated (black arrow), and the hepatic plate atrophy disappeared. D Central hepatic vein occlusion and congestion, the branches fibrosis and occlusion, thickened its wall and disordered hepatic plate arrangement (black arrow) 

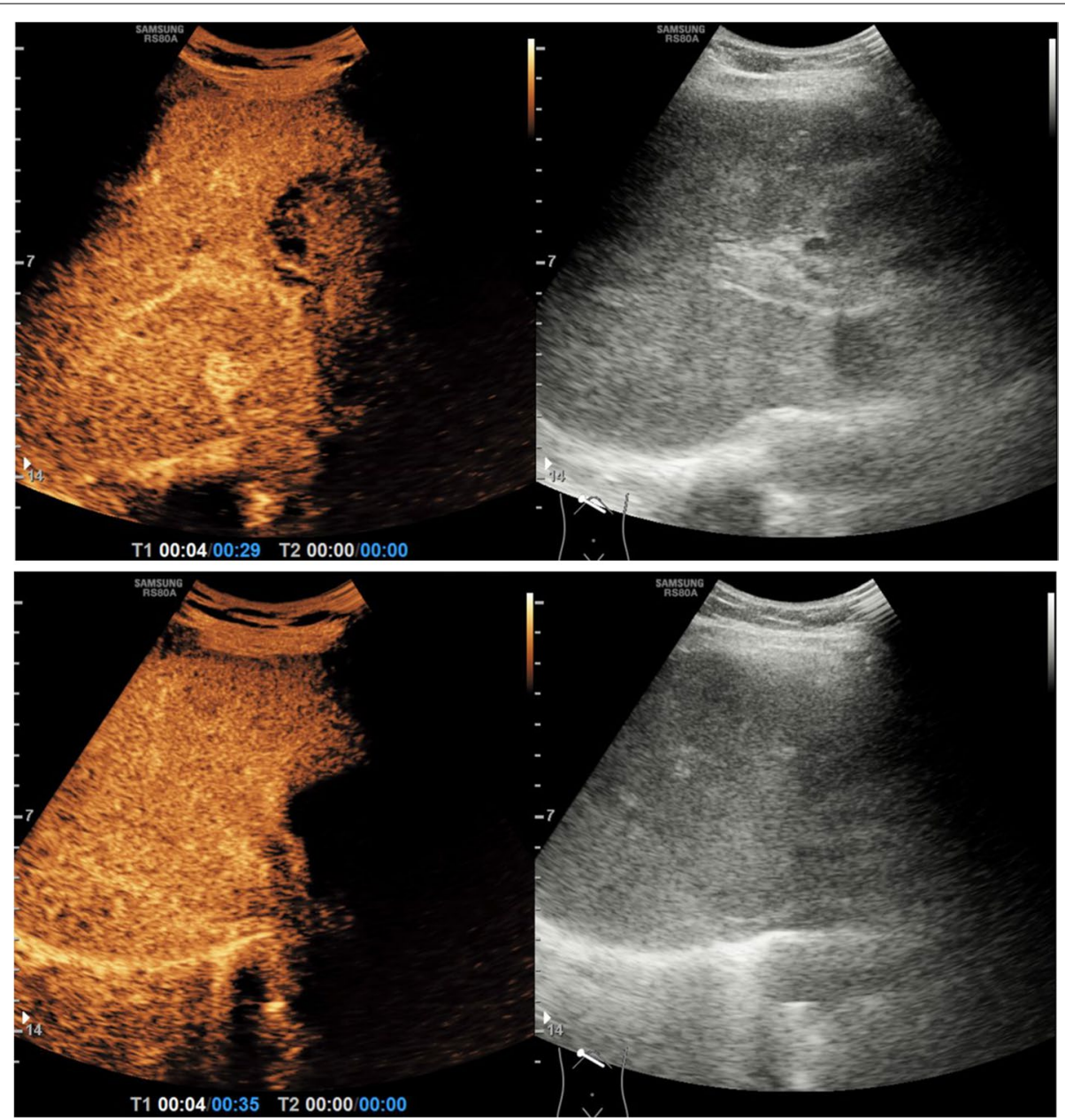

Fig. 3 Contrast enhanced ultrasonography after acute liver failure. The echo of liver parenchyma is not uniform, attributed to the high pressure of the hepatic sinusoid. Portal vein perfusion was slightly delayed, there was no hepatic artery-portal vein fistula, and no thrombosis was found in the portal vein

mixture of aspartic acid, ornithine, and arginine were administered.

To identify the etiology of ALF, detailed medical history of the patient was recorded by the patient and her family, whereby they admitted that the patient had taken the GS (Tusanqi) $2-3$ g every $24 \mathrm{~h}$ for one and a half years. A liver biopsy was conducted for diagnosis. CD34 immunostaining of liver biopsy $(200 \times)$ revealed that positive CD34 was present in the subendothelial space of central veins and perivenular zones (Fig. 2B). Hematoxylin-eosin staining of liver biopsy $(200 \times)$ results showed highly dilated hepatic sinusoid, disappeared hepatic plate atrophy and some portal inflammatory (Fig. 2C). Further, the central venous lumen was irregular and became smaller, the branches of which were fibrosis and occlusion, and its wall was thickened (Fig. 2D). Biopsy results, laboratory findings, and clinical course confirmed the diagnosis of DILI.
Five days after the operation, an emergency contrastenhanced ultrasonography confirmed a slight delay in the portal vein perfusion phase, absence of hepatic arteryportal vein fistula, and absence of thrombosis in the portal vein (Fig. 3). Broadly heterogeneous hypo-attenuating areas observed by abdominal CT indicated massive liver necrosis (Fig. 4A). The patient was in a shallow coma. The patient underwent one session of Double plasma molecular absorb system treatment, an ALSS, combined with symptomatic treatment. Following therapy, significant improvement was noted in her clinical signs and symptoms, which was substantiated by the laboratory parameters (Table 1), and follow-up CT also demonstrated that the degree of liver injury was greatly alleviated, the heterogeneous hypoattenuating areas disappeared, and liver volume also recovered (Fig. 4B). Finally, in the third week post operation, the patient got discharged from the hospital and followed up at the outpatient clinic. The patient 


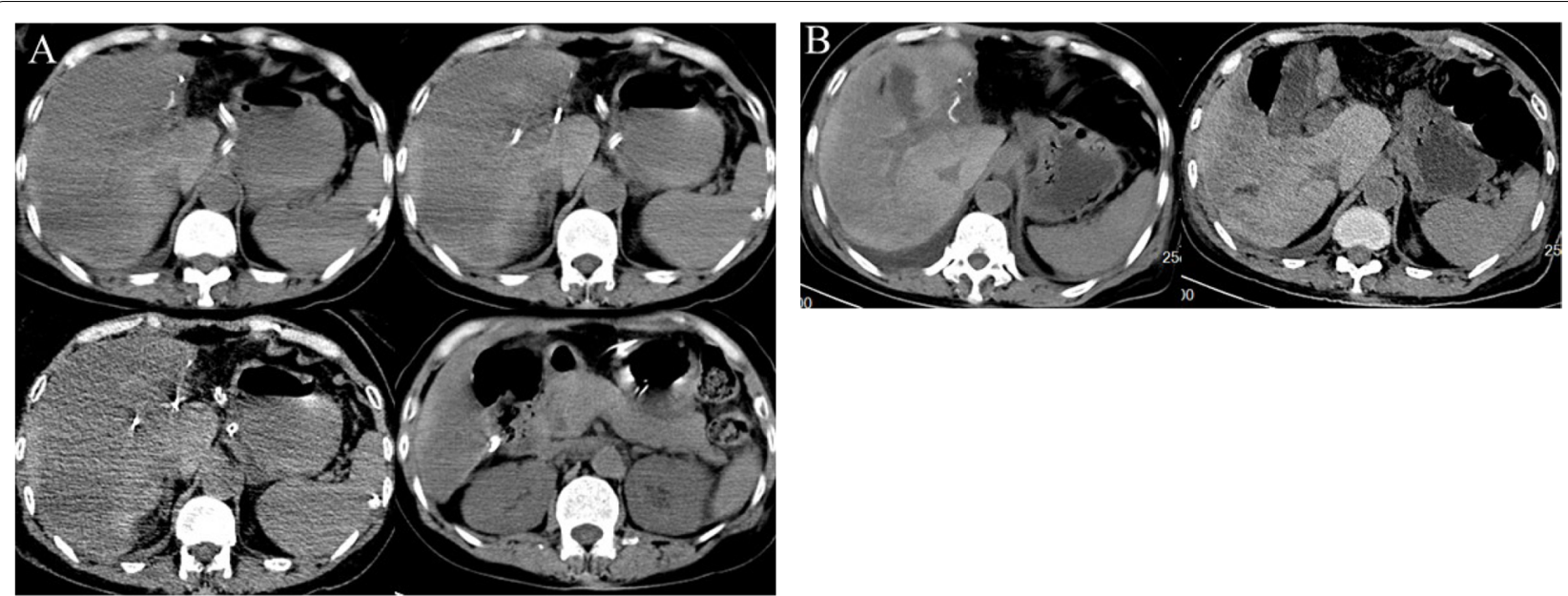

Fig. 4 A Emergency abdominal CT showing marked liver atrophy and broadly heterogeneous hypoattenuating areas implied heterogeneous hepatic necrosis. B Fourteen days after the operation, follow-up CT claimed the disappearance of the heterogeneous hypoattenuating areas coupled with the recovery of the patient's liver volume

was in a generally good condition, and no recurrence of DILI and development of liver cirrhosis was reported as of the last follow-up visit at two years after the discharge.

\section{Discussion and conclusions}

DILI is referred to as the liver injury resulting from all types of prescription or non-prescription drugs, including Nonsteroidal Anti-inflammatory Drugs, antimicrobials, Traditional Chinese medicines, natural medicines, health products, and dietary supplements [4-6]. It is often encountered in clinical practice with an incidence of 14-19 cases per 100,000 per year in developed countries, the acute DILI accounts for about $20 \%$ of hospitalized patients with acute liver injury [7].

In China, 38 Chinese herbal medicines contain pyrrolizidine alkaloids (PAs), with GS being the most widely used. Two hepatotoxic PAs, namely senecionine and seneciphylline, were indentified in GS by using the HPLC-UV-MS analytical method [1]. These PAs are metabolic activated in the liver through CYP (P450 cytochrome) to electrophilic pyrrolic metabolites. The reactive metabolites generated in situ react with hepatocellular proteins. The resulting protein adduction causes hepatocyte damage by a variety of genotoxicities, including DNA binding, DNA cross-linking, DNA-protein cross-linking, sister chromatid exchange, chromosomal aberrations, mutagenicity, teratogenicity, and carcinogenicity [8]. Furthermore, PAs can decrease glutathione (GSH) in sinusoidal endothelial cells (SEC). This enhanced oxidative stress also can activate hepatic stellate cells [9]. Another study suggested that the main manifestations of PA-induced liver toxicity were progressive damage to SEC, loss of all cell types that comprise the sinusoidal wall, severe central vein $(\mathrm{CV})$ endothelial cells' damage, decreased numbers of Kupffer cells in the lobule, recruitment of monocytes into the lobule and adherent to the $\mathrm{CV}$ endothelium, subendothelial and advential fibrosis of CVs, and damage of the CV endothelium with subendothelial hemorrhage [10]. Interestingly, the observed pyrrole-protein adducts in the blood of rats treated with GS appeared to gradually vanish from 100\% (day 1) to $64.5 \%$ (week 1), 15.2\% (week 2), 10.7\% (week 3 ), and undetectable (week 4) after dosing [1]. Thus, only long-term consumption of GS can cause DILI, finally leading to HSOS or liver cirrhosis.

Based on the course of disease, DILI is classified into acute DILI and chronic DILI, the diagnostic criteria of chronic DILI should meet the following conditions: within 6 months after DILI occurs, serum ALT, AST, ALP, or TBIL still remain abnormal, or there is radiographic and histological evidence for portal hypertension or chronic liver injury [11]. The clinical manifestations of acute DILI are usually non-specific. The incubation period varies greatly across individuals, ranging from a few days to several months, and mostly depends on the dose and duration of consumption of the drugs. Most patients with DILI may have no significant symptoms and only manifest the liver biochemical indexes increased in varying degrees such as serum ALT, AST, TBIL, DBIL and ALP [11]. Detection of DILI is more challenging when the patient is suffering from chronic liver diseases. Atypical symptoms and signs of liver injury and portal hypertension may be attributed by patients and clinicians to chronic liver disease, not DILI.

In the absence of any specific clinical characteristics and biological markers of DILI, the diagnostic strategy 
mainly adopts the DILI algorithm recently proposed by ACG in combination with the Council for International Organizations of Medical Sciences-Roussel Uclaf Causality Assessment Method (RUCAM) [5, 12]. In our case, according to the DILI algorithm recommended by ACG, initial screening with hepatitis serologies, autoimmune serologies, imaging, and liver biopsy were accomplished. Compiling all the evaluations, the viral and autoimmune hepatitis, hepatic ischemia and metabolic disorders were reasonably excluded. Application of the RUCAM to our case estimated the patient's RUCAM score as 6 (1: Drug start $>90$ days, 2: Decrease $\geq 50 \%$ in 30 days, 1 : Age $>55$, 2: Reaction in product label) [5], which indicated a "probable" relationship with GS. These findings collectively helped to confirm a diagnosis of DILI caused by GS.

ALF is a rare and severe consequence of abrupt hepatocyte injury, resulting in a consistent pattern of rapidonset elevation of aminotransferases, coagulopathy and encephalopathy within a short period of time [13]. Idiosyncratic DILI is the second most common cause of ALF after paracetamol [14]. Only 10\% of all patients with DILI develop ALF, with the potential for liver transplantation or death. When combined with chronic liver disease, once DILI occurs there is a higher risk for the appearance of ALF or even death. While the occurrence of ALF in DILI is mainly associated with drug-induced hepatotoxicity and aggravating inducing factors, including surgery $[15,16]$.

Identification of GS or other factors as the reason for ALF, in this case, is a quite complex issue because abnormal liver enzymes occur after partial hepatectomy; several factors were involved and must be taken into consideration. However, the following aspects presume that ALF in our patient was not caused by underlying diseases and surgery. First, the patient had no history of drinking and was found to be negative for the serological tests for infectious hepatitis, HIV, or other viruses, and had no history of chronic portal hypertension. Autoimmune diseases were also excluded. It is considered that the other liver diseases and hypoxic hepatitis possess less propensity to develop ALF. Meanwhile, intraoperative exploration found that intrahepatic bile duct stones were mainly involved in the left lateral lobe of the liver, the common bile duct and the right liver were not affected by atrophy and obstruction. There was no abnormal liver function before operation. Therefore, it is not considered that chronic left localized hepatolithiasis can affect the function of the right liver and leads to ALF. Moreover, surgery is a big trigger, not a cause. During the operation, the blood flow into the liver was blocked two times for a total of $35 \mathrm{~min}$ with the help of the Pringle operation, satisfying the safe occlusion time of $120 \mathrm{~min}$ [17]. The possibility of hepatic ischemia resulting from the intraoperative amputation of the hepatic pedicle and the formation of intrahepatic venous thrombosis can be effectively ruled out by conducting postoperative contrast-enhanced ultrasonography (Fig. 3). Only the diseased left external lobe of the patient's liver was removed, which was well below the safe limits of FLR after liver resection (30\% to $50 \%$ of the future liver remnant (FLR)/CT-based nontumor liver volume or formula-based liver volume) [18]. After excluding other potential etiologies, GS-induced DILI was the most probable cause of ALF in this case.

For this patient, she was a 63 years old woman. She had been taking GS (2-3 g once per day) for a year and a half, and have primary hepatolithiasis for more than six years. Therefore, she might have higher risk for the appearance of ALF. In the current case, consumption of pyrrolizidine alkaloids-containing GS is prone to develop chronic DILI, manifested as HOSO, which is associated with hepatic megalocytosis, ascites, hyperbilirubinaemia, and hemorrhagic necrosis [19]. The current case is the first report in which GS induces acute DILI, developing the most serious form of DILI, namely ALF. In addition, Her clinical features and prognosis were different from GS related HSOS, which presented jaundice, right upper quadrant pain, portal hypertension, slow progress towards ALF and eventually led to liver cirrhosis [2].

In conclusion, physicians should be aware of the potential liver toxicity of GS. In case of patients with a history of taking GS and other hepatotoxic drugs, strict vigilance should be paid to DILI. Liver biopsy remains the important method for the diagnosis of DILI. Preoperative investigation of the reserve capacity and compensatory capacity of liver function for patients with DILI could avoid postoperative ALF as far as possible. For the treatment of DILI-induced ALF, the drug should be withheld instantly, symptomatic treatment, ALSS and liver transplantation should be actively implemented.

\section{Abbreviations \\ DILI: Drug-induced liver injury; ALF: Acute liver failure; HSOS: Hepatic sinusoid obstruction syndrome; ALSS: Artificial liver support system; CT: Computed tomography; HIV: Human immunodeficiency virus; MRI: Magnetic Reso- nance Imaging; POD: Postoperative Day; WBC: White blood cell count; HGB: Hemoglobin; PLT: Platelets; hsCRP: High sensitivity C-reactive protein; AST: Aspartate aminotransferase; ALT: Alanine aminotransferase; TBIL: Total bilirubin; DBIL: Direct bilirubin; IBIL: Indirect bilirubin; LDH: Lactate dehydrogenase; ALP: Alkaline phosphatase; ALB: Albumin; Glu: Glucose; PT: Prothrombin time.}

\section{Acknowledgements}

We would like to thank Dr. Gang Xu of Department of pathology, Sichuan Academy of Medical Sciences \& Sichuan Provincial People's Hospital for his interpretation of pathological findings.

\section{Authors' contributions}

ZLZ and HBZ wrote the manuscript under the close supervision of XLH; ZLZ, DZL and JS collected patient's data and contributed to the text of the manuscript; SNS provided histopathology figures and description; QYY and YS contributed to technical support and design of the research, GMX, YTY, THF 
and DYZ managed the patient, CYL and YS provided final edits and expert opinion in the field. All authors approved the final manuscript.

\section{Funding}

This study was supported by the National Basic Research Program of, the National Institute of China (\#2015CB964703), Scientific and Technological Transformative Project of Sichuan Scientific Research Institutions (\# 14010127: \#2017QN08), and Project of Science \& Technology Department of Sichuan Province (\# 2016JY0020; \# 2018HH0062), Sichuan Science and Technology Program (\# 2021YFH0187)

\section{Availability of data and materials}

All data generated or analysed during this study are included in this published article.

\section{Declarations}

Ethics approval and consent to participate

This study was approved by the Sichuan Academy of Medical Sciences \& Sichuan Provincial People's Hospital ethics committee.

\section{Consent for publication}

Written informed consent was obtained from the patient for publication of this case report. A copy of the written consent and operation contract are available for review by the editor of this journal.

\section{Competing of interests}

The authors declare that they have no competing interests.

\section{Author details}

'Department of Hepatobiliary-Pancreatic Surgery, Cell Transplantation Center, Sichuan Provincial People's Hospital, University of Electronic Science and Technology of China, Chengdu, China. ${ }^{2}$ Chinese Academy of Sciences Sichuan Translational Medicine Research Hospital, Chengdu, China. ${ }^{3}$ Department of Pathology, Sichuan Provincial People's Hospital, University of Electronic Science and Technology of China, Chengdu, China.

Received: 28 June 2021 Accepted: 2 March 2022

Published online: 08 March 2022

\section{References}

1. Lin G, Wang JY, Li N, Li M, Gao H, Ji Y, Zhang F, Wang H, Zhou Y, Ye Y, et al. Hepatic sinusoidal obstruction syndrome associated with consumption of Gynura segetum. J Hepatol. 2011;54(4):666-73.

2. Ou P, Liu X, Tang Z, Hou Z, Liu L, Liu J, Zhou S, Fang Z, Sun K, Chen Y, et al. Gynura Segetum related hepatic sinusoidal obstruction syndrome: a liver disease with high mortality and misdiagnosis rate. Curr Pharm Des. 2019;25(35):3762-8.

3. Wang JY, Gao H. Tusanqi and hepatic sinusoidal obstruction syndrome. $J$ Dig Dis. 2014;15(3):105-7.

4. Bjornsson ES, Bergmann OM, Bjornsson HK, Kvaran RB, Olafsson S. Incidence, presentation, and outcomes in patients with drug-induced liver injury in the general population of Iceland. Gastroenterology. 2013;144(7):1419-25 1425 e1411-1413; quiz e1419-1420.

5. Chalasani NP, Hayashi PH, Bonkovsky HL, Navarro VJ, Lee WM, Fontana RJ. Practice Parameters Committee of the American College of G: ACG Clinical Guideline: the diagnosis and management of idiosyncratic druginduced liver injury. Am J Gastroenterol. 2014;109(7):950-66 quiz 967.

6. Devarbhavi H. An update on drug-induced liver injury. J Clin Exp Hepatol. 2012;2(3):247-59.

7. Hoofnagle JH, Longo DL, Björnsson ES. Drug-induced liver injury — types and phenotypes. N Engl J Med. 2019;381(3):264-73.

8. Fu PP, Xia Q, Lin G, Chou MW. Pyrrolizidine alkaloids-genotoxicity, metabolism enzymes, metabolic activation, and mechanisms. Drug Metab Rev. 2004;36(1):1-55.

9. Wang XKG, DeLeve LD. Support of sinusoidal endothelial cell glutathione prevents hepatic veno-occlusive disease in the rat. Hepatology. 2000:31:428-34.
10. DeLeve LDMR, Wang X, Hu L, McCuskey MK, Epstein RB, Kanel G. Characterization of a reproducible rat model of hepatic veno-occlusive disease. Hepatology. 1999;29:1779-91.

11. Yu YC, Mao YM, Chen CW, Chen JJ, Chen J, Cong WM, Ding Y, Duan ZP, Fu QC, Guo XY, et al. CSH guidelines for the diagnosis and treatment of drug-induced liver injury. Hepatol Int. 2017;11(3):221-41.

12. Agarwal VK, McHutchison JG, Hoofnagle JH. Drug-Induced Liver Injury $\mathrm{N}$ : Important elements for the diagnosis of drug-induced liver injury. Clin Gastroenterol Hepatol. 2010:8(5):463-70.

13. Stravitz RT, Lee WM. Acute liver failure. Lancet 2019;394(10201):869-81.

14. Reuben A, Tillman H, Fontana RJ, Davern T, McGuire B, Stravitz RT, Durkalski V, Larson AM, Liou I, Fix O, et al. Outcomes in adults with acute liver failure between 1998 and 2013: an observational cohort study. Ann Intern Med. 2016;164(11):724-32.

15 Reuben A, Koch DG, Lee WM. Acute Liver Failure Study G: Drug-induced acute liver failure: results of a U.S. multicenter, prospective study. Hepatology. 2010;52(6):2065-76.

16. Wei G, Bergquist A, Broomé U, Lindgren S, Wallerstedt S, Almer S, Sangfelt P, Danielsson Å, Sandberg-Gertzén H, Lööf L, et al. Acute liver failure in Sweden: etiology and outcome. J Intern Med. 2007;262(3):393-401.

17. van Riel WG, van Golen RF, Reiniers MJ, Heger M, van GulikTM. How much ischemia can the liver tolerate during resection? Hepatobiliary Surg Nutr. 2016;5(1):58-71.

18. Kawaguchi Y, Lillemoe HA, Vauthey JN. Dealing with an insufficient future liver remnant: portal vein embolization and two-stage hepatectomy. J Surg Oncol. 2019;119(5):594-603.

19. Helmy A. Review article: updates in the pathogenesis and therapy of hepatic sinusoidal obstruction syndrome. Aliment Pharmacol Ther. 2006:23(1):11-25.

\section{Publisher's Note}

Springer Nature remains neutral with regard to jurisdictional claims in published maps and institutional affiliations.

Ready to submit your research? Choose BMC and benefit from:

- fast, convenient online submission

- thorough peer review by experienced researchers in your field

- rapid publication on acceptance

- support for research data, including large and complex data types

- gold Open Access which fosters wider collaboration and increased citations

- maximum visibility for your research: over $100 \mathrm{M}$ website views per year

At BMC, research is always in progress.

Learn more biomedcentral.com/submissions 